CERN-PPE/93-123

15 July 1993

\title{
IDENTIFIED PARTICLE INTERFEROMETRY IN HEAVY-ION COLLISONS: RESULTS FROM THE CERN NA-44 EXPERIMENT
}

\author{
H. Beker ${ }^{\mathrm{a}, 1}$, H. Bøggild ${ }^{\mathrm{b}}$, J. Boissevain ${ }^{\mathrm{c}}$, M. Cherney ${ }^{\mathrm{d}}$, J. Dodd ${ }^{\mathrm{e}}$, S. Esumif ${ }^{\mathrm{f}}$, \\ C.W. Fabjan ${ }^{\mathrm{a}}$, D. Fields ${ }^{\mathrm{c}}$, A. Franz ${ }^{\mathrm{a}}$, K.H. Hansen ${ }^{\mathrm{b}}$, B. Holzer ${ }^{\mathrm{a}}$, T. Humanic ${ }^{\mathrm{g}}$, \\ B. Jacak ${ }^{c}$, R. Jayanti ${ }^{\text {h }}$ H. Kalechofsky ${ }^{\text {h }}$, T. Kobayashi, ${ }^{\mathrm{i}, 2}$, R. Kvatadze ${ }^{\mathrm{a}, 3}$, Y.Y. Lee $^{\mathrm{h}}$, \\ M. Leltchouk ${ }^{\mathrm{e}}$, B. Lorstad ${ }^{\mathrm{j}}$, N. Maeda ${ }^{\mathrm{f}}$, A. Medvedeve ${ }^{\mathrm{e}}$ A. Miyabayashi ${ }^{\mathrm{j}}$, M. Murrayk $^{\mathrm{k}}$, \\ S. Nagamiya ${ }^{\mathrm{e}}$, S. Nishimura ${ }^{\mathrm{f}}$, S.U. Pandey ${ }^{\mathrm{h}}$, F. Piuz ${ }^{\mathrm{a}}$, V. Polychronakos ${ }^{\mathrm{l}}$, M. Potekhin ${ }^{\mathrm{e}}$, \\ G. Poulard ${ }^{\mathrm{a}}$, A. Sakaguchi ${ }^{\mathrm{f}}$, M. Sarabura ${ }^{\mathrm{c}}$, K. Shigaki ${ }^{\mathrm{a}, 5}$, J. Simon-Gilloc ${ }^{c}$, W. Sondheim ${ }^{c}$, \\ T. Sugitate ${ }^{f}$, J. Sullivan ${ }^{c}$, Y. Sumi ${ }^{f}$, H. van Hecke ${ }^{c}$, W.J. Willis ${ }^{\mathrm{e}}$ and K. Wolf ${ }^{\mathrm{k}}$
}

\begin{abstract}
A large sample of $\pi$ and $\mathrm{K}$ pairs, produced in $\mathrm{S}-\mathrm{Pb}$ and $\mathrm{p}-\mathrm{Pb}$ collisions has been studied. Results of a 3-dimensional correlation function analysis are presented. We observe significant differences between radii measured with pion and kaon pairs.
\end{abstract}

Submitted to the International Europhysics Conference,

Marseille, July 1993.

a CERN, CH-1211 Geneva 23, Switzerland

b Niels Bohr Institute, DK-2100 Copenhagen, Denmark

${ }^{c}$ Los Alamos National Laboratory, Los Alamos, NM 87545, USA

d Creighton University, Omaha, NE, USA

e Columbia University, New York, NY 10027, USA

f Hiroshima University, Higashi-Hiroshima 724, Japan

$g$ Ohio State University, Columbus, OH 43210, USA

h University of Pittsburgh, Pittsburgh, PA 15260, USA

i National Laboratory for High Energy Physics, Tsukuba 305, Japan

j University of Lund, S-22362 Lund, Sweden

k Texas A+M University, College Station, TX 77843, USA

${ }^{1}$ Brookhaven National Laboratory, Upton, NY 11973, USA

1 Present address: Rome I University, Rome I-00185, Italy

${ }^{2}$ Present address: Riken Linac Laboratory, Riken, Saitama 35101, Japan

3 Visitor from Tbilisi State University, Tbilisi, Georgia

${ }^{4}$ Present address: Tsukuba University, Tsukuba 305, Japan

5 University of Tokyo 113, Japan 

Two-particle intensity interferometry can provide information on the space-time extent of the particle-emitting source [1]. Correlation measurements with identified particles may be particularly helpful in understanding the dynamical evolution of heavy-ion collisions. It has also been argued that such measurements may provide evidence for the existence of a first-order phase transition in such collisions [2].

NA44 is a second-generation experiment, optimized for the study of single- and two-particle distributions [3]. The spectrometer ('focusing' spectrometer) is designed to have good acceptance for pairs of particles with small momentum difference at midrapidity. The apparatus also has excellent momentum resolution $(\delta \mathrm{p} / \mathrm{p} \sim 0.2 \%)$ and allows the study of particle distributions over a wide $\mathrm{p}_{\mathrm{T}}$ range: $0.0 \lesssim \mathrm{p}_{\mathrm{T}} \lesssim 1.5 \mathrm{GeV} / \mathrm{c}$. We obtain large data samples with small statistical and systematic errors, such that multidimensional analyses with identified hadrons (pions, kaons, protons) become possible. In this contribution we present recent results on identified two-particle correlations.

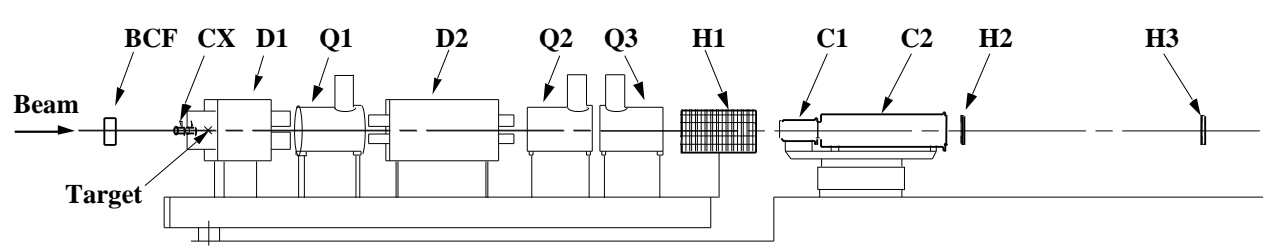

Figure 1: Schematic layout of the NA44 experimental set-up

\section{$2 \quad$ Experimental set-up}

The spectrometer (Fig. 1) uses two dipole magnets (D1, D2) for momentum dispersions; three superconducting quadrupoles (Q1, Q2, Q3) of large aperture provide the focusing over a relatively large phase space $(\sim \pm 20 \% \Delta \mathrm{p}$ around nominal momentum setting). Scintillator hodoscopes (H1, H2, H3) of high granularity are used for tracking in the momentum analysis and provide time-of-flight (TOF) information at the $\sigma \approx 100 \mathrm{ps}$ level. Time of flight together with threshold-Cherenkov information (C1, C2) provides the basis for particle identification (Fig. 2).

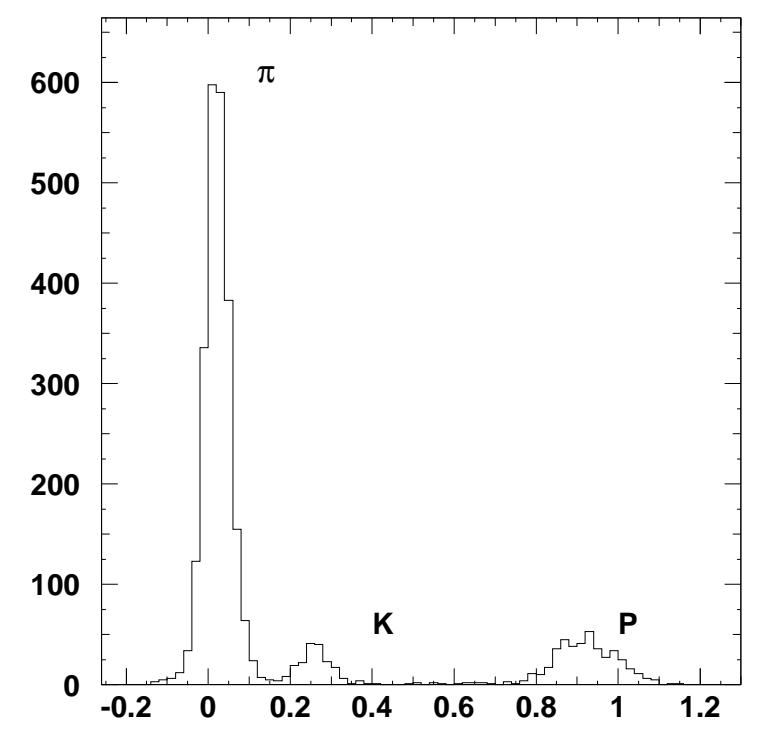

Figure 2: Observed time-of-flight distributions as a function of $\mathrm{m}^{2}$ 
The spectrometer is operated at different nominal momentum settings such that a complete coverage in $\mathrm{p}_{\mathrm{T}}$ can be achieved. We operate the spectrometer also at two different angles (44 mrad and $122 \mathrm{mrad}$ ) to map the rapidity- $\mathrm{p}_{\mathrm{T}}$ space. Typical acceptances are displayed in Fig. 3.

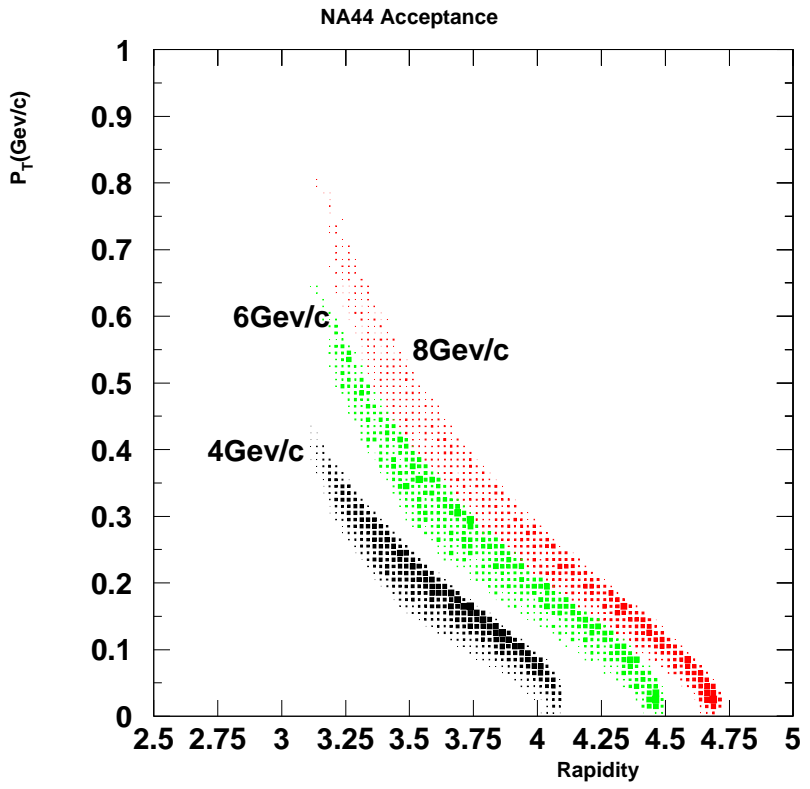

Figure 3: The single-particle reponse for pions in $\mathrm{y}$ and $\mathrm{p}_{\mathrm{T}}$ for three different spectrometer settings (44 mrad operation). The $\mathrm{p}_{\mathrm{T}}$-range is increased by operating the spectrometry at larger angles $(122 \mathrm{mrad})$

A multiplicity trigger is used to select central collisions, typically at the level of $10 \%$ of the total inelastic cross-section.

\section{Data analysis}

We present preliminary results on several data sets [4], as summarized in Table 1.

Table 1

Data sample used in interferometry analysis

\begin{tabular}{|cccc|}
\hline Collision & $\begin{array}{c}\text { Particles } \\
\text { analysed }\end{array}$ & $\begin{array}{c}\text { Spectrometer } \\
\text { setting }\end{array}$ & $\begin{array}{c}\text { Sample } \\
\text { size }\end{array}$ \\
\hline $\mathrm{p}(450 \mathrm{GeV} / \mathrm{c})+\mathrm{Pb}$ & $\pi^{+} \pi^{+}$ & $4 \mathrm{GeV} / \mathrm{c}$ & $400 \mathrm{k}$ \\
& $\mathrm{K}^{+} \mathrm{K}^{+}$ & $6 \mathrm{GeV} / \mathrm{c}$ & $5 \mathrm{k}$ \\
$\mathrm{S}(200 \mathrm{GeV} / \mathrm{c} \cdot \mathrm{n})+\mathrm{Pb}$ & $\pi^{+} \pi^{+}$ & $4 \mathrm{GeV} / \mathrm{c}$ & \\
& & $44 \mathrm{mrad}$ & $320 \mathrm{k}$ \\
& & $120 \mathrm{mrad}$ & $100 \mathrm{k}$ \\
& $\mathrm{K}^{+} \mathrm{K}^{+}$ & $6 \mathrm{GeV} / \mathrm{c}$ & $60 \mathrm{k}$ \\
& $\mathrm{K}^{-} \mathrm{K}^{-}$ & $6 \mathrm{GeV} / \mathrm{c}$ & $6 \mathrm{k}$ \\
\hline
\end{tabular}

Based on this data sample the raw correlation function can be constructed:

$$
\mathrm{C}_{\mathrm{raw}}\left(\mathrm{k}_{1}, \mathrm{k}_{2}\right)=\mathrm{R}\left(\mathrm{k}_{1}, \mathrm{k}_{2}\right) / \mathrm{B}\left(\mathrm{k}_{1}, \mathrm{k}_{2}\right),
$$

where $\mathrm{k}_{1}, \mathrm{k}_{2}$ are the particle momenta. The distribution $\mathrm{R}\left(\mathrm{k}_{1}, \mathrm{k}_{2}\right)$ denotes the measured distribution of the relative momentum and $\mathrm{B}\left(\mathrm{k}_{1}, \mathrm{k}_{2}\right)$ the 'background' distribution. The 
background distribution or normalization distribution should ideally be constructed from the inclusive, single-particle distribution. We have, however, used 'event' mixing of the pair sample to avoid an impact parameter bias and have corrected the single-particle spectra for the distortion (due to the correlation and the Coulomb interaction) in the pair spectrum [3,5].

In addition to the corrections to the single-particle spectrum, $\mathrm{K}_{\mathrm{SPC}}\left(\mathrm{k}_{1}, \mathrm{k}_{2}\right)$, effects due to particle acceptance $K_{a c c}\left(k_{1}, k_{2}\right)$ and the Coulomb interaction $K_{\text {coul }}\left(k_{1}, k_{2}\right)$ (we use both the method of Coulomb wave-function integration [6] and Gamow corrections) need to be considered to obtain the physical correlation function [3]:

$$
\mathrm{C}\left(\mathrm{k}_{1}, \mathrm{k}_{2}\right)=\mathrm{C}_{\mathrm{raw}} \cdot \mathrm{K}_{\mathrm{SPC}} \cdot \mathrm{K}_{\mathrm{acc}} \cdot \mathrm{K}_{\mathrm{coul}} \cdot
$$

The experimental results are usually summarized in a more compact form by fitting the correlation function to one of the following forms, with the relevant definitions explained in Fig. 4:

a)

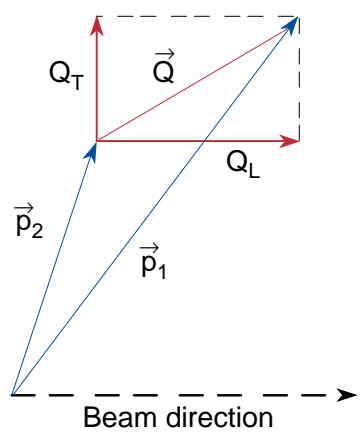

b)

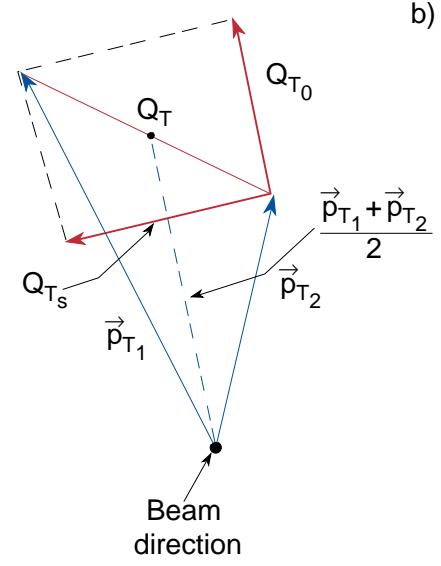

Figure 4: Coordinate system and definition of the various components of the momentum difference, used in the analysis

1) $\mathrm{C}\left(\mathrm{Q}_{\text {inv }}\right)=1+\lambda \exp \left[-\mathrm{Q}_{\text {inv }}^{2} \mathrm{R}_{\text {inv }}^{2}\right]$,

2) $\mathrm{C}\left(\sqrt{\mathrm{Q}^{2}+\mathrm{Q}_{0}^{2}}\right)=1+\lambda \exp \left[-\left(\mathrm{Q}^{2}+\mathrm{Q}_{0}^{2}\right) \mathrm{R}^{2}\right]$,

3) $\quad \mathrm{C}\left(\mathrm{Q}_{\mathrm{T}_{0}}, \mathrm{Q}_{\mathrm{T}_{S}}, \mathrm{Q}_{\mathrm{L}}\right)=1+\lambda \exp \left[-\mathrm{Q}_{0}^{2} \mathrm{R}_{\mathrm{T}_{0}}^{2}-\mathrm{Q}_{\mathrm{T}_{S}}^{2} \mathrm{R}_{\mathrm{T}_{S}}^{2}-\mathrm{Q}_{\mathrm{L}}^{2} \mathrm{R}_{\mathrm{L}}^{2}\right]$,

where

$$
\mathrm{Q}=\left|\overrightarrow{\mathrm{p}}_{1}-\overrightarrow{\mathrm{p}}_{2}\right|, \quad \mathrm{Q}_{0}=\left|\mathrm{E}_{1}-\mathrm{E}_{2}\right|, \quad \mathrm{Q}_{\mathrm{inv}}^{2}=\mathrm{Q}^{2}-\mathrm{Q}_{0}^{2} .
$$

$\mathrm{C}\left(\mathrm{Q}_{\text {inv }}\right)$ has been frequently used in the past and we have published pion-pair correlations, using this parametrization [3]. However, the physics interpretation of the radius parameter extracted from $\mathrm{C}\left(\mathrm{Q}_{\text {inv }}\right)$ is ambiguous, particularly when comparing different particles. The analysis in terms of $\mathrm{C}\left(\sqrt{\mathrm{Q}^{2}+\mathrm{Q}_{0}^{2}}\right)$ is presented here, mainly to permit comparison with correlation results obtained with similar nuclear systems, but at the much lower AGS energies $\left(\mathrm{E}_{\text {beam }}=14.6 \mathrm{GeV} / \mathrm{c} \cdot \mathrm{n}\right)[6]$. Our high-statistics data permit also a 3-dimensional analysis $\mathrm{C}\left(\mathrm{Q}_{\mathrm{T}_{0}}, \mathrm{Q}_{\mathrm{T}_{\mathrm{S}}}, \mathrm{Q}_{\mathrm{L}}\right)$, which may provide the most valuable insight into the physics. In particular, it has been argued [2] that 3-dimensional measurements may discriminate a first-order phase transition to a quark-gluon plasma from a dense hadron 
gas. In the latter, the fast hadronization results in $\mathrm{R}_{\mathrm{T}_{\mathrm{S}}} \approx \mathrm{R}_{\mathrm{T}_{0}}$. In the former, characterized by considerable latent energy, the hadronization is much slower and we expect $R_{T_{0}}>R_{T_{\S}}$.

Our results are summarized in Table 2. The quality of our data can be judged from one example, 3-dimensional $\mathrm{K}^{+} \mathrm{K}^{+}$correlation functions, as shown in Fig. 5. It should be noted that the data are Gamow-corrected and preliminary; the errors quoted in the table are statistical only. Systematic errors have been evaluated [3] and are found to be smaller or at most comparable with the statistical errors.

Table 2

Summary on radii obtained from fits to NA44 data (preliminary)

\begin{tabular}{|cccccc|}
\hline System & Part. & $\mathrm{R}=\tau$ & $\mathrm{R}_{\mathrm{T}_{0}}$ & $\mathrm{R}_{\mathrm{T}_{\mathrm{S}}}$ & $\mathrm{R}_{\mathrm{L}}$ \\
\hline $\mathrm{S}-\mathrm{Pb}$ & $\pi^{+}$ & $4.0 \pm 0.1$ & $4.1 \pm 0.1$ & $4.4 \pm 0.2$ & $4.9 \pm 0.3$ \\
$(200 \mathrm{GeV} / \mathrm{n})$ & & & & & \\
$"$ & $\pi^{-}$ & $3.9 \pm 0.1$ & $3.6 \pm 0.2$ & $3.7 \pm 0.5$ & $4.4 \pm 0.4$ \\
$"$ & $\mathrm{~K}^{+}$ & $2.7 \pm 0.1$ & $2.7 \pm 0.2$ & $2.4 \pm 0.3$ & $2.8 \pm 0.3$ \\
$"$ & $\mathrm{~K}^{-}$ & $2.8 \pm 0.3$ & $3.2 \pm 0.4$ & - & $3.6 \pm 0.6$ \\
$\mathrm{p}+\mathrm{Pb}$ & $\pi^{+}$ & $2.1 \pm 0.1$ & $1.8 \pm 0.1$ & $1.4 \pm 0.1$ & $2.5 \pm 0.1$ \\
$(450 \mathrm{GeV})$ & & & & & \\
$"$ & $\mathrm{~K}^{+}$ & $1.5 \pm 0.2$ & $1.9 \pm 0.2$ & - & $3.3 \pm 0.5$ \\
$\mathrm{~S}+\mathrm{Pb}$ & $\pi^{+}$ & $2.1 \pm 0.1$ & - & - & - \\
$\left(\left\langle\mathrm{p}_{\mathrm{T}}\right\rangle \sim 0.5 \mathrm{GeV} / \mathrm{c}\right)$ & & & & & \\
\hline
\end{tabular}
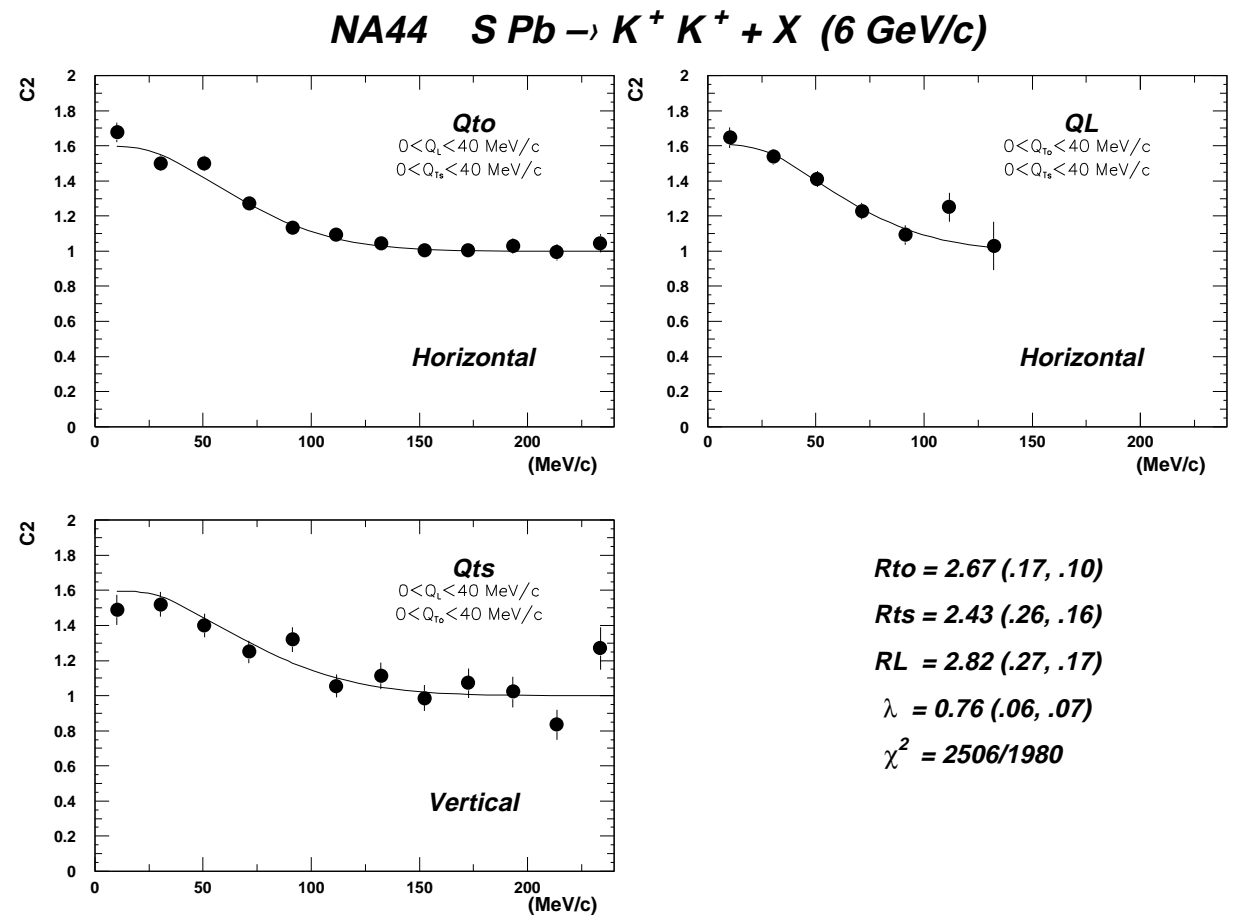
Rto $=2.67(.17, .10)$
Rts $=2.43(.26, .16)$
$R L=2.82(.27, .17)$
$\lambda=0.76(.06, .07)$
$\chi^{2}=2506 / 1980$

Figure 5: Three-dimensional analysis of the $\mathrm{K}^{+} \mathrm{K}^{+}$data sample (see text for explanation) 


\section{Discussion of Results}

AGS data.

In Fig. 6a we summarize the $\mathrm{R}=\tau$ fits and compare them with the lower-energy

We find a significant difference in the observed radii, depending on the particles observed $\left(\mathrm{R}_{\pi^{+} \pi^{+}} \approx \mathrm{R}_{\pi^{-} \pi^{-}}>\mathrm{R}_{\mathrm{KK}}\right)$. The radii for equivalent systems measured by the E802 Collaboration are significantly lower than our data. It should be noted that the $y$ and $\mathrm{p}_{\mathrm{T}}$ acceptances of the two experiments are quite comparable and are not expected to be the origin of this difference. The energy density $\epsilon$ created in these central collisions is however much larger in the NA44 data; we consider this comparison as a striking indication that these nuclear systems expand to larger dimensions with increasing energy density of the collision system.

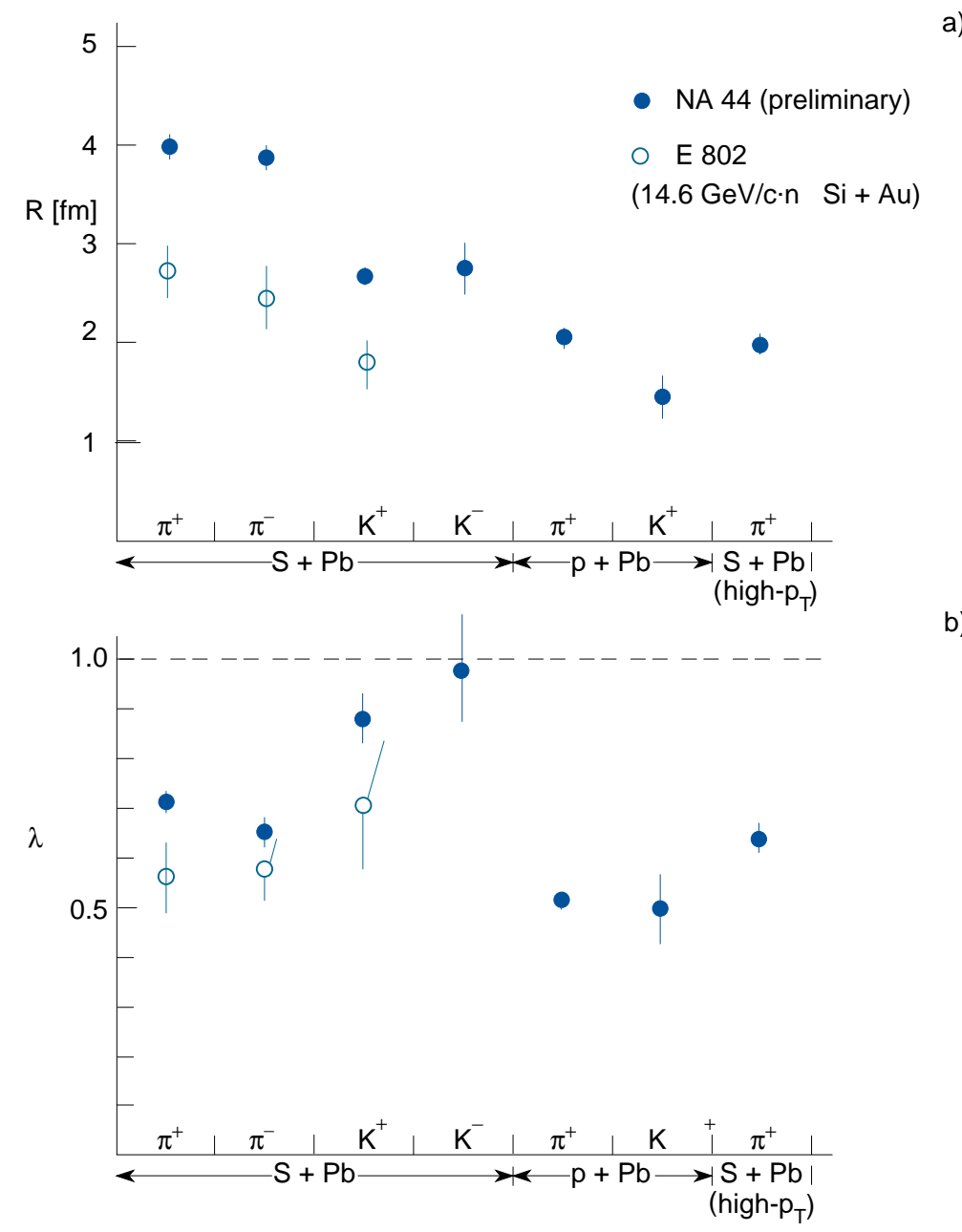

Figure 6: a) Summary of the $\mathrm{R}=\tau$ analysis for different systems and comparison with the E802 data; b) summary of the results on the chaoticity parameter $\lambda$ for different systems

The comparison of the chaoticity parameter $\lambda$, Fig. $6 \mathrm{~b}$, is also interesting. The pion correlations show $\lambda \sim 0.6$ to $0.7<1$; this result is usually attributed to the dilution of the correlation strength due to the presence of pions from resonance decays $(\omega$, and in particular $\left.\eta, \eta^{\prime}, \ldots\right)$. Resonance contributions $(\phi)$ are estimated to be much smaller in the KK system, and indeed we find $\lambda \approx 1$ ! The equality of radii measured with $\mathrm{K}^{+}$and $\mathrm{K}^{-}$ indicates that hyperon resonances are not important.

Further insight may be gained by comparing our results with estimates based on 
the picture that the bosons are emitted at 'freeze-out' from a spherical volume with a freeze-out radius $\mathrm{R}_{\mathrm{FO}} \sim$ mean free path $\lambda[7]$. Results of such an evaluation are compared with $\mathrm{R}_{\mathrm{T}_{S}}$ in Fig. 7 . Rather good agreement is found with the measurements. It is also instructive to realize that the radius of the projectile (Gaussian equivalent) is considerably smaller than the radii observed in our correlation analysis. In a picture, where the volume of the projectile is filled with non-interacting pions, the Gaussian equivalent radius would be

$$
\mathrm{R}_{\text {projectile }}(\text { Gaussian equiv. })=\frac{1}{\sqrt{5}} 1.2 \mathrm{~A}_{\text {projectile }}^{1 / 3},
$$

which we have used for this comparison with $\mathrm{R}_{\mathrm{T}_{\mathrm{S}}}$. This radius was chosen because it best measures the geometrical size of the source.

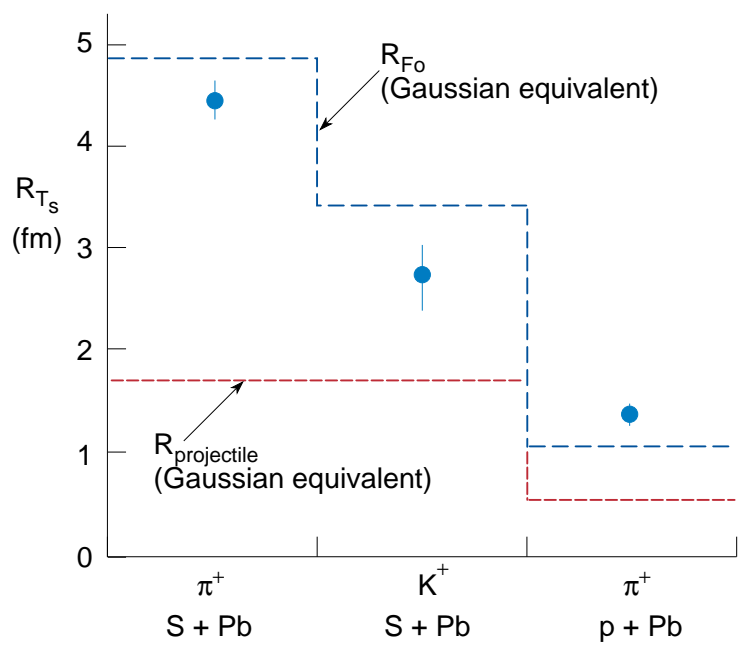

Figure 7: Comparison of the data on $\mathrm{R}_{\mathrm{T}_{\mathrm{S}}}$ with the radii $\mathrm{R}_{\mathrm{F}_{0}}$ estimated from a simple 'freeze-out' model. The nuclear projectile radius is also indicated (see text)

We also note that the source radius extracted from the high- $\mathrm{p}_{\mathrm{T}} \pi^{+}$sample gives a significantly smaller value compared to the lower- $_{\mathrm{T}}$ data obtained in the small-angle exposure. This effect is under study using event generators, such as RQMD [8].

\section{$5 \quad$ Conclusions}

The high-statistics data of identified boson pairs obtained in $\mathrm{S}-\mathrm{Pb}$ collisions may be summarized as follows:

$$
\begin{aligned}
& \left.\begin{array}{l}
\mathrm{R}_{\pi^{+}} \sim \mathrm{R}_{\pi^{-}} \sim 4 \mathrm{fm} \\
\mathrm{R}_{\mathrm{K}^{+}} \sim \mathrm{R}_{\mathrm{K}^{-}} \sim 3 \mathrm{fm}
\end{array}\right\} \mathrm{S}+\mathrm{Pb} \\
& \lambda_{\pi}<\lambda_{\mathrm{K}} \sim 1 \\
& \mathrm{R}_{\mathrm{T}_{0}} \approx \mathrm{R}_{\mathrm{T}_{S}} \\
& \mathrm{R}_{\pi} \gtrsim 2 \mathrm{R}_{\text {projectile }} \\
& \mathrm{R}(200 \mathrm{GeV} / \mathrm{c} \cdot \mathrm{n})>\mathrm{R}(14.6 \mathrm{GeV} / \mathrm{c} \cdot \mathrm{n}) \text {. }
\end{aligned}
$$

Available NA44 data from $\mathrm{S}+\mathrm{Ag}, \mathrm{S}+\mathrm{S}, \mathrm{p}+\mathrm{S}, \mathrm{p}+$ Be (correlation data and single inclusive spectra) should provide further insight into the dynamics of these dense nuclear systems. Comparisons with event generators, such as RQMD, provide important tools for the analysis of these collisions. 


\section{Acknowledgements}

The NA44 Collaboration wishes to thank the staff of the CERN PS-SPS accelerator complex for their excellent work. We thank the technical staff at CERN and the collaborating insitutes for their valuable contributions. We are also grateful for the support given by the Austrian Science Foundation; the Science Research Council of Denmark; the Japanese Society for the Promotion of Science and the Ministry of Education, Science and Culture, Japan; the Science Research Council of Sweden; the US Department of Energy; and the National Science Foundation (Nuclear Physics) through grants PHY8906284 and PHY8958491.

\section{References}

[1] B. Lorstad, Int. J. Mod. Phys. A4 (1988) 2861;

N.A. Zajc, A pedestrian's guide to interferometry, to be published in Proc. NATO Advanced Study Institute on Particle Production in Highly Excited Matter, Lucca, Italy, eds. H. Gutbrod and J. Rafelski (1992).

[2] G. Bertsch and G.E. Brown, Phys. Rev. C4 (1989) 320.

[3] H. Beker et al., Phys. Lett. B302 (1993) 510.

[4] A. Franz, New results on HBT and single particle spectra from NA44, to be published in Proc. XXVII Rencontre de Moriond, 1993.

[5] K. Kadija and P. Seyboth, Phys. Lett. B287 (1992) 362.

[6] T. Abbott et al., Phys. Rev. Lett. 69 (1992) 1030.

[7] T. Humanic, Invited presentation at the Quark Matter '93 Conference, to be published in the Proceedings.

[8] J.P. Sullivan et al., Phys. Rev. Lett. 70 (1993) 3000. 